\section{Manejo de la sepsis basada en evidencias para países} en vías de desarrollo

\section{Sepsis evidence based management for developing countries}

\section{Sr. Editor:}

La medicina basada en evidencias (MBE) se sustenta en el uso de la mejor evidencia científica para la toma de las mejores decisiones en el cuidado del paciente, que se realiza utilizando los diversos recursos de MBE, por lo que el manejo de toda patología debe basarse en ella, con especial atención en las condiciones asociadas a alta morbimortalidad como la sepsis.

La sepsis es una condición clínica descrita hace más de dos siglos, definida en consensos desde 1991 como el resultado de un síndrome de respuesta inflamatoria sistémica a un proceso infeccioso, y que recientemente planteó un cambio en su definición, siendo considerada una disfunción orgánica que pone en riesgo la vida debido a una respuesta irregular del hospedero a la infección ${ }^{1}$. La nueva definición ha generado una gran discusión, suscitando modificaciones en diversos aspectos como la inclusión del score quickSOFA (qSOFA), o versión rápida de la evaluación de falla orgánica relacionada con sepsis (SOFA: Sepsis-related Organ Failure Assessment).

El qSOFA permite una valoración netamente clínica sin la necesidad de análisis de laboratorio, con el fin de identificar y tratar tempranamente a los pacientes con sepsis; y cuya aplicación en los servicios de emergencia y cuidados críticos es fácil de realizar en países en vías de desarrollo, pues solo incluye parámetros clínicos que permiten un diagnóstico temprano de la enfermedad. La aplicación de esta nueva definición en los países en desarrollo podría permitir una disminución en la morbimortalidad asociada.

En Latinoamérica, la incidencia de sepsis se ha incrementado en los últimos años, y se ha acompañado de nuevos estudios disponibles a nivel mundial para el diagnóstico temprano (qSOFA), manejo y prevención de la sepsis. Actualmente la información disponible enfocada hacia su uso en países en desarrollo es escasa, contando con sólo una publicación desarrollada hace 4 años para un manejo global de la sepsis ${ }^{2}$, y una publicación reciente de recomendaciones específicas para el manejo de la infección ${ }^{3}$. Es por este motivo que, en los países en desarrollo, y especialmente en los latinoamericanos, es necesario realizar un manejo de la sepsis basado en evidencias.

En los últimos años se han desarrollado escasas guías a nivel mundial para el manejo de la sepsis, siendo la de la campaña de supervivencia (2013) la más utilizada internacionalmente. Esta guía ha sido adecuadamente implementada a nivel mundial y en Latinoamérica a través del Instituto Latinoamericano de sepsis; sin embargo, su utilidad actual podría estar limitada por la constante nueva evidencia disponible ${ }^{4}$, siendo necesaria a la fecha disponer de GPC actualizadas, considerando que éstas deben ser actualizadas en promedio en un período de 2 años desde su publicación ${ }^{5}$.

En los países en desarrollo, la aplicación de las GPC disponibles para el manejo de la sepsis es escasa y dificultosa, debido principalmente al acceso variable, largas distancias a los hospitales, capacidad de triaje, y diagnóstico retardado ${ }^{6}$. Ante esta realidad, y con el fin de lograr un manejo adecuado de la sepsis, es necesario que las sociedades latinoamericanas o grupos de trabajo realicen procesos de adaptación de las GPC disponibles (Tabla 1), que serán publicadas pronto y que incluirán las nuevas definiciones y/o estudios publicados.

Tabla 1. Guías de práctica clínica para el manejo de la sepsis

\begin{tabular}{|c|c|c|c|c|c|c|}
\hline Guía de práctica clínica & Tipo & País & Idioma & $\begin{array}{l}\text { Organización } \\
\text { desarrolladora }\end{array}$ & $\begin{array}{c}\text { Fecha de } \\
\text { publicación* }\end{array}$ & Estado \\
\hline Sepsis & De novo & $\begin{array}{l}\text { Reino } \\
\text { Unido }\end{array}$ & Inglés & NICE & $\begin{array}{l}13 \text { de julio } \\
\text { de } 2016\end{array}$ & Publicada \\
\hline $\begin{array}{l}\text { Prevención, diagnóstico, } \\
\text { terapia y seguimiento } \\
\text { de sepsis }\end{array}$ & De novo & Alemania & Alemán & AWMF & $\begin{array}{l}31 \text { de marzo } \\
\text { de } 2017\end{array}$ & $\begin{array}{l}\text { Bajo } \\
\text { revisión }\end{array}$ \\
\hline $\begin{array}{l}\text { Campaña de supervivencia } \\
\text { de la Sepsis: Guía Internacional } \\
\text { para el manejo de la sepsis } \\
\text { grave y el shock séptico }\end{array}$ & De novo & Internacional & $\begin{array}{l}\text { Español/ } \\
\text { inglés }\end{array}$ & $\begin{array}{l}\text { Comité de recomenda- } \\
\text { ciones de la campaña } \\
\text { para sobrevivir a la } \\
\text { sepsis, que incluye el } \\
\text { subgrupo de pediatría }\end{array}$ & $\begin{array}{l}30 \text { de enero } \\
\text { de } 2013\end{array}$ & Publicada \\
\hline
\end{tabular}

Fuente: Guidelines International Network. www.g-i-n.net. NICE: National Institute for Health and Care Excellence. AWMF: Association of Scientific Medical Societies. 
La adaptación se puede realizar a través de diversos métodos disponibles como el ADAPTE o RAPADAPTE, que inician con el planteamiento de preguntas y desenlaces "outcomes" específicos para la realidad donde se plantea adaptar. En los países en desarrollo, algunas de las preguntas más importantes a ser abordadas son: ¿Cuándo un paciente séptico requiere un catéter venoso central? ¿Qué tipo de fluidos se debe utilizar para la reanimación en el paciente séptico? ¿Cómo se deben administrar los vasopresores en el servicio de emergencia (vía central o periférica)? ¿Cuándo se deben administrar fármacos inotrópicos?, entre otras. Entre los "outcomes" más importantes se encuentran: Disminución de mortalidad, estancia hospitalaria, eventos adversos, y secuelas; medición del lactato sérico, de saturación venosa central para inicio de inotrópicos, de diferencia arterio-venosa de $\mathrm{CO}_{2}$, y de marcadores de seguimiento en sepsis.

El siguiente paso es evaluar la calidad metodológica de las GPC actualizadas y que se encuentren disponibles a texto completo, con el instrumento AGREE II (Appraisal of Guidelines Research and Evaluation), con el fin de seleccionar aquellas que obtengan un puntaje mínimo necesario y sean adaptadas para el contexto local. Estas evaluaciones frecuentes en diversas patologías, no ha sido realizado para las GPC de sepsis.

Por lo mencionado anteriormente, tener un manejo basado en evidencias para el manejo de sepsis en países en desarrollo supone un reto que nos invita a evaluar, adaptar, e implementar GPC actualizadas periódicamente, que permitan estar al día con el avance científico mundial, siendo una responsabilidad de las diversas sociedades y o grupos de trabajo disponibles sobre el tema.

\section{José Gálvez-Olortegui ${ }^{1,2}$, Carlos Plasencia-Meza ${ }^{3,4,5}$, Lucía Castro-Pinedo ${ }^{3,5}$, José Urcia-Vazallo ${ }^{3,6}$, Magno Gonzáles-Soto ${ }^{3,7}$, María Hinojosa-Méndez ${ }^{3,4,7}$, Tomas Gálvez-Olortegui ${ }^{1,8}$ \\ ${ }^{1}$ Unidad Generadora de Evidencias y Vigilancia Epidemiológica, Scientia Clinical and Epidemiological Research Institute, Trujillo, Perú. \\ ${ }^{2}$ Facultad de Medicina-Universidad Nacional de Trujillo, Trujillo, Perú. \\ ${ }^{3}$ Grupo de Trabajo en Sepsis, Scientia Clinical and Epidemiological Research Institute, Trujillo, Perú. \\ ${ }^{4}$ Facultad de Medicina-Universidad Privada Antenor Orrego, Trujillo, Perú. \\ ${ }^{5}$ Departamento de Emergencia-Hospital Regional Docente de Trujillo, Trujillo, Perú.}

${ }^{6}$ Departamento de Cuidados Críticos-Hospital Victor Lazarte Echegaray, Trujillo, Perú.

${ }^{7}$ Departamento de Emergencia-Instituto Regional de Enfermedades Neoplásicas, Trujillo, Perú.

${ }^{8}$ Escuela de Posgrado-Universidad Privada Antenor Orrego, Trujillo, Perú.

\section{Referencias}

1. Opal SM, Rubenfeld GD, Poll T Van Der, Vincent J, Angus DC. The Third International Consensus Definitions for Sepsis and Septic Shock (Sepsis-3). JAMA. 2016; 315 (8): 801-10.

2. Dünser MW, Festic E, Dondorp A, Kissoon N, Ganbat T, Kwizera A, et al. Recommendations for sepsis management in resource-limited settings. Intensive Care Med 2012; 38 (4): 557-74.

3. Thwaites CL, Lundeg G, Dondorp AM, sepsis in resource-limited settings-expert consensus recommendations group of the European Society of Intensive Care Medicine (ESICM) and the Mahidol-Oxford Research Unit (MORU) in Bangkok T. Recommendations for infection management in patients with sepsis and septic shock in resource-limited settings. Intensive Care Med 2016; 21; 4-6.

4. Deutschman CS, Neli. Evidence Based Practice of Critical Care. 2nd ed. Philadelphia, USA: Elsevier; 2016. 614 p.

5. Shekelle PG, Ortiz E, Rhodes S, Morton SC, Eccles MP, Grimshaw JM, et al. Validity of the Agency for Healthcare Research and Quality clinical practice guidelines: how quickly do guidelines become outdated? JAMA 2001; 286 (12): 1461-7.

6. Jacob ST, Lim M, Banura P, Bhagwanjee S, Bion J, Cheng $\mathrm{AC}$, et al. Integrating sepsis management recommendations into clinical care guidelines for district hospitals in resource-limited settings: the necessity to augment new guidelines with future research. BMC Med 2013; 11: 107.

Conflictos de interés: Los autores declaran no tener ningún conflicto de intereses.

Correspondencia a: José Gálvez-Olortegui Scientia Clinical and Epidemiological Research Institute. Mz. G Lt. 22 Urb. Vista Hermosa-Trujillo-Perú.

Teléfono: +51985079008

jgalvezo@scientiaceri.com 